\title{
A sparse mapping of structure to function in microbial communities
}

Summary: Simple models quantitatively predict metabolite dynamics in denitrifying bacterial communities from gene content alone.

$$
\text { Karna Gowda, }{ }^{1,2} \text { Derek Ping, }{ }^{3} \text { Madhav Mani, }{ }^{4,5,6 *} \text { Seppe Kuehn }{ }^{1,2 *}
$$

${ }^{1}$ Department of Ecology and Evolution, University of Chicago, Chicago, IL 60637, USA

${ }^{2}$ Center for the Physics of Evolving Systems, University of Chicago, Chicago, IL 60637, USA

${ }^{3}$ Department of Physics, University of Illinois at Urbana-Champaign, Urbana, IL 61801, USA

${ }^{4}$ Department of Engineering Sciences and Applied Mathematics, Northwestern University, Evanston, IL 60208, USA

${ }^{5}$ Department of Molecular Biosciences, Northwestern University, Evanston, IL 60208, USA

${ }^{6}$ NSF-Simons Center for Quantitative Biology, Northwestern University, Northwestern University, Evanston, IL 60208, USA

*To whom correspondence should be addressed;

E-mail: madhav.mani@gmail.com, seppe.kuehn@gmail.com. 
The metabolic function of microbial communities emerges through a complex hierarchy of genome-encoded processes, from gene expression to interactions between diverse taxa. Therefore, a central challenge for microbial ecology is deciphering how genomic structure determines metabolic function in communities. Here we show, for the process of denitrification, that community metabolism is quantitatively predicted from the genes each member of the community possesses. Quantifying metabolite dynamics across a diverse library of bacterial isolates enables a statistical approach that reveals a sparse mapping from gene content to metabolic phenotypes. A consumer-resource model then correctly predicts community metabolism from the metabolic phenotypes of each strain. Our results enable connecting metagenomic data to metabolite dynamics, designing denitrifying communities, and discovering how genome evolution impacts metabolism.

\section{Introduction}

Microbial metabolism plays an essential role in sustaining life on Earth. Working collectively in complex communities, microbes are key players in global nutrient cycles (1), wastewater treatment (2) and human health (3). As such, a key challenge in microbial ecology is understanding how emergent community metabolism is determined by the taxonomic and genomic structure of a community. Addressing this structure-function problem is critical for functionally interpreting community gene content (4), elucidating the evolutionary principles of community metabolism $(5,6)$ and designing synthetic communities (7).

Understanding how community metabolism is genomically encoded requires mapping the genotypes of each community member to metabolic phenotypes, and then deciphering how interactions between distinct populations contribute to collective metabolism. Quantitatively mapping genotypes to metabolic phenotypes for naturally-occurring bacteria is challenging due to substantial genetic and phenotypic variation in the wild (8). Moreover, interactions within communities depend on extracellular metabolites (9), abiotic factors (10), cooperation (11) and higher-order effects $(12,13)$. While constraint-based models have found some success in predicting collective metabolism from genomes (14-16), these methods require significant manual refinement (17), complicating the prospect of making predictions from the genomes of nonmodel organisms or metagenomes of communities.

Despite these apparent obstacles, recent studies hint that gene content may be strongly predictive of emergent metabolic function at the community-level. First, sequencing studies of diverse microbiomes show that, while abundances of individual taxa can be highly variable (18), the genes or pathways a community possesses are stable across communities in similar environments $(18,19)$, suggesting that gene content might be a conserved feature of complex communities (20). Additional studies have extended this insight by demonstrating that the functional gene content of a community correlates with local metabolite concentrations (21-23). In the lab- 
oratory, enrichment experiments have revealed that diverse taxa harboring conserved metabolic phenotypes are assembled to degrade exogenously supplied organic carbon $(24,25)$, but the quantitative relationship between gene content and metabolic phenotypes in these contexts is not yet clear.

One route to mapping gene content to metabolite dynamics is to quantify the flux of metabolites through an ensemble of genomically-diverse communities, and then take a statistical approach to predict metabolite fluxes from genomes. Natural communities harbor sufficient genomic diversity for this approach, but quantifying metabolite dynamics in situ is challenging in most contexts (19). Experiments in the laboratory can overcome these obstacles, but previous studies either have focused only on abundance dynamics $(25,26)$ or utilized model organisms with limited genomic diversity (7). These considerations underscore the necessity for making quantitative measurements of metabolite dynamics using diverse communities in the laboratory to relate structure and function.

Here we took a statistical approach to mapping gene content to metabolite dynamics using a diverse ensemble of bacterial isolates. We measured the dynamics of metabolite consumption and production for each isolate, and parameterized these dynamics using a simple consumerresource model. The genomic diversity of the ensemble of isolates enabled a regression approach to mapping gene content to consumer-resource model parameters, which resulted in a sparse mapping of genomic structure to metabolic phenotypes. Finally, the consumer-resource model captured interactions between strains mediated by resources, yielding predictions for community-level metabolite dynamics, which we verified experimentally.

\section{Results}

\section{Denitrification as a model metabolic process}

We used denitrification as a model metabolic process (Fig. 1A) because it is performed by diverse, culturable bacterial taxa, it is well-characterized at the molecular level, it is a collective process, and the relevant metabolites are readily quantifiable (27). Denitrification is a form of anaerobic respiration whereby microbes use oxidized nitrogen compounds as electron acceptors, driving a cascade of four successive reduction reactions, $\mathrm{NO}_{3}^{-} \rightarrow \mathrm{NO}_{2}^{-} \rightarrow \mathrm{NO} \rightarrow \mathrm{N}_{2} \mathrm{O} \rightarrow$ $\mathrm{N}_{2}$ (27). As a biogeochemical process, denitrification is essential to nitrogen cycling at a global scale through activity in soils, freshwater systems, and marine environments (28), and impacts human health through activity in wastewater treatment plants (2) and in the human gut (29). The process is performed by taxonomically-diverse bacteria (30) that are typically facultative anaerobes. The denitrification pathway is known to be modular, with some strains performing all four steps in the cascade, and others performing one or a nearly arbitrary subset of reduction reactions (31). Denitrification in nature is therefore a collective process, where a given strain can produce electron acceptors that can be utilized by other strains (9).

We focused experimentally on the first two steps of denitrification: the conversion of nitrate 
A

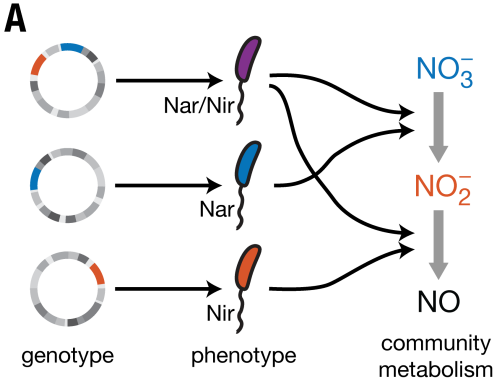

B

B ACM04 (Nar/Nir)

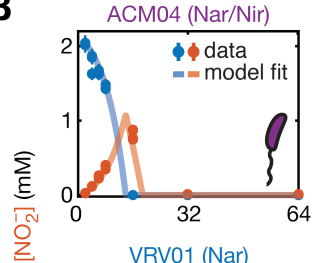

है

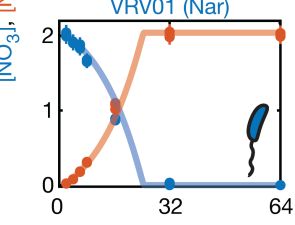

C

$$
\begin{aligned}
\frac{d x}{d t} & =\left(\gamma_{A} r_{A} \frac{A}{K_{A}+A}+\gamma_{I} r_{I} \frac{I}{K_{I}+I}\right) x \\
\frac{d A}{d t} & =-r_{A} \frac{A}{K_{A}+A} x \\
\frac{d I}{d t} & =\left(r_{A} \frac{A}{K_{A}+A}-r_{I} \frac{I}{K_{I}+I}\right) x
\end{aligned}
$$

D

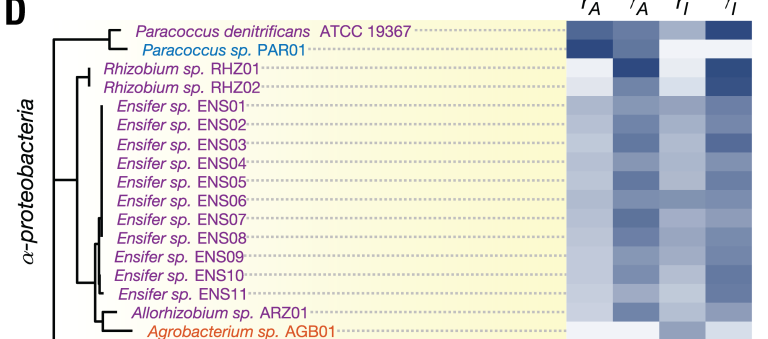

Figure 1: Quantifying nitrate and nitrite dynamics in an ensemble of denitrifiers to map genomic structure to metabolic function. (A) A roadmap for relating genomic structure to community metabolic function across a diverse library of bacteria. First, mapping the genotypes (circles) of individual strains to their metabolic phenotypes (straight arrows) and then combining the metabolic activity of each strain in a community to predict collective metabolite dynamics $\left(\mathrm{NO}_{3}^{-}, \mathrm{NO}_{2}^{-}\right.$, right). (B) Example batch culture metabolite dynamics for Nar/Nir (purple), Nar (blue), and $\mathrm{Nir}$ (red) isolates. Nitrate $\left(\mathrm{NO}_{3}^{-}\right.$, blue points) and nitrite $\left(\mathrm{NO}_{2}^{-}\right.$, red points) dynamics are measured at logarithmically-spaced intervals (circles) via sampling and colorimetric assay (see Materials and Methods). Abundances are only measured at the final time point. Curves show fits to a consumer-resource model shown in panel C. (C). A consumerresource model of nitrate and nitrite reduction by each strain describes abundances $(x)$, nitrate concentration $(A)$, and nitrite concentration $(I)$ in time. The model is parameterized by reduction rates $r_{A}$ and $r_{I}$ and yields $\gamma_{A}$ and $\gamma_{I}$, for growth on nitrate and nitrite respectively. The affinity parameters $\left(K_{*}\right)$ were not well-constrained by the data and were fixed for all strains in the library (see Supplementary Text). (D) Phylogenetic tree and normalized consumer-resource parameters for 62 denitrifying strains (61 isolates and the model denitrifier Paracoccus denitrificans). Phylogenetic tree constructed using the 16S rRNA gene, and scale bar indicates the estimated number of substitutions per site. Darker colors indicate larger values of the normalized parameters. Each isolate was assigned a unique identifier. Phenotypic parameters measured across diverse isolates constitutes a dataset for relating genomic diversity to metabolite dynamics. 
$\left(\mathrm{NO}_{3}^{-}\right)$to nitrite $\left(\mathrm{NO}_{2}^{-}\right)$and subsequently nitric oxide (NO) (Fig. 1A). Nitrate and nitrite are soluble, enabling high throughput measurements of metabolite dynamics. Rather than working only with model organisms, we isolated 61 diverse bacterial strains spanning $\alpha-, \beta-$, and $\gamma$-proteobacteria from local soils using established techniques (Materials and Methods). Each strain was obtained in axenic culture and was characterized as performing one or both of the first two steps of denitrification in a chemically-defined, electron acceptor-limited medium containing a single non-fermentable carbon source (succinate). Each of these strains was therefore classified into one of three possible phenotypes (Fig. 1A): (1) Nar/Nir strains that perform both nitrate and nitrite reduction $\left(\mathrm{NO}_{3}^{-} \rightarrow \mathrm{NO}_{2}^{-} \rightarrow \mathrm{NO}\right)$, (2) Nar strains that perform only nitrate reduction $\left(\mathrm{NO}_{3}^{-} \rightarrow \mathrm{NO}_{2}^{-}\right.$), and (3) Nir strains that perform only nitrite reduction $\left(\mathrm{NO}_{2}^{-} \rightarrow \mathrm{NO}\right)$. In addition to these 61 isolates, our strain library also included the model Nar/Nir strain Paracoccus denitrificans (ATCC 19367).

\section{Parameterizing metabolite dynamics}

We first set out to quantify the metabolic phenotypes of each isolate in our diverse strain library. We focused our efforts on quantifying the dynamics of the relevant metabolites, nitrate and nitrite. To accomplish this, strains were inoculated at low starting densities into 96-well plates containing chemically-defined medium with either nitrate or nitrite provided as the sole electron acceptor, and then incubated under anaerobic conditions. Small samples $(10 \mu \mathrm{L})$ were then taken at logarithmically-spaced time intervals over a period of $64 \mathrm{~h}$ and assayed for nitrate and nitrite concentrations (Materials and Methods, Fig. S1 and S2). At the end of the time course, optical density was assayed. The measurement resulted in a time series of nitrate and nitrite production/consumption dynamics in batch culture (points, Fig. 1B).

To parameterize the metabolite dynamics of each strain within a common framework, we utilized a consumer-resource model, which explicitly relates the growth of each strain to the dynamics of metabolite production and consumption (Fig. 1C). For each strain in monoculture, we parameterized the consumer-resource model using measured denitrification dynamics across a range of initial cell densities and nitrate/nitrite concentrations (Supplementary Text, Fig. S3 and S4). The model allowed us to quantitatively describe the phenotype of each strain in the library using at most four parameters: $r_{A}$ and $r_{I}$, which capture rates of nitrate and nitrite reduction, and $\gamma_{A}$ and $\gamma_{I}$, which describe yields for nitrate and nitrite, respectively. Substrate affinities $\left(K_{*}\right)$ were fixed to a small value since these parameters were not well constrained by the data (Supplementary Text, Fig. S5). The models for Nar and Nir strains correspond to setting $r_{I}=0$ or $r_{A}=0$, respectively. Yields $\left(\gamma_{*}\right)$ were inferred using endpoint optical density measurements, and rates $\left(r_{*}\right)$ were inferred by fitting the observed nitrate and nitrite dynamics to the consumer-resource model (Fig. 1C). Remarkably, with the exception of a small number of strains that were excluded from the library (Supplementary Text, Fig. S6), a single set of parameters quantitatively described metabolite dynamics for each strain across a range of initial cell densities and nitrate/nitrite concentrations (Supplementary Text, Fig. S4).

Fitting our consumer-resource model to data for each strain yielded a quantitative descrip- 
tion of the dynamic metabolic phenotype of each strain in the library (Fig. 1B and D). We observed large variability between taxa, with coefficients of variation for both rates $\left(r_{A}, r_{I}\right)$ and yields $\left(\gamma_{A}, \gamma_{I}\right)$ around $60 \%$. We also observed some patterns of phylogenetic conservation, for example $\alpha$-proteobacteria produced generally higher yields than $\beta$ - or $\gamma$-proteobacteria, and a clade of Pseudomonas sp. isolates showed consistently higher rates of nitrite reduction than most other strains (Fig. 1D). Despite these patterns, the prevalence of each of the three phenotypes is not strongly dependent on phylogeny, with each phenotype present across the tree (Fig. 1D). The latter observation is consistent with pervasive horizontal gene transfer of denitrifying enzymes $(32,33)$. Finally, we did not observe a trade-off between rates and yields (Fig. S7).

\section{Predicting metabolite dynamics from genomes}

The rates and yields measured across our library of isolates (Fig. 1D) enabled us to take a statistical approach to relating genome structure to measured metabolic phenotypes. Motivated by the observation that gene content correlates strongly with environmental variation at the community-level (23), and that the statistics of gene presence and absence capture functional information (34), we used a simple machine learning approach to predict consumer-resource model parameters from gene presence/absence alone. To accomplish this, we performed whole genome sequencing on all 62 strains in the library. Then we assembled and annotated each genome (Materials and Methods) and determined the complement of denitrification genes possessed by each strain, exploiting the fact that the molecular and genetic basis of denitrification is well-understood (27). We identified not only the reductases that perform the reduction of the oxidized nitrogen compounds, but also the sensors/regulators (35) and transporters (36) known to be involved in denitrification (Materials and Methods). The presence and absence of each gene (or set of genes encoding proteins that form a complex) in each genome is presented in Fig. 2A. Patterns of gene presence/absence agree well with known features of the denitrification pathway, including the mutual exclusion of the two reductases performing nitrite reduction (NirS and NirK) (21,33)). Further, in almost all cases strains possessing nitrate and/or nitrite reductase performed the associated reactions in culture (with the only exception being the Nar strain Acidovorax sp. ACV01, which possesses both nitrate and nitrite reductase). This is in agreement with previous work demonstrating that bacterial genomes lose nonfunctional genes due to streamlining (37).

Next we showed that the presence and absence of denitrification genes in each strain was sufficient to quantitatively predict metabolite dynamics in monoculture. Specifically, we constructed a linear regression where the measured phenotypic parameters of our consumer-resource model were predicted on the basis of gene presence and absence (Fig. 2B). The regression coefficients for each gene in the pathway quantify the impact of the presence of the gene on a given phenotypic parameter. We used $L_{1}$-regularized regression (least absolute shrinkage and selection operator, LASSO) to avoid overfitting (Supplementary Text, Fig. S8 to S10), performing independent regressions for each of the phenotypic parameters in our consumer-resource 


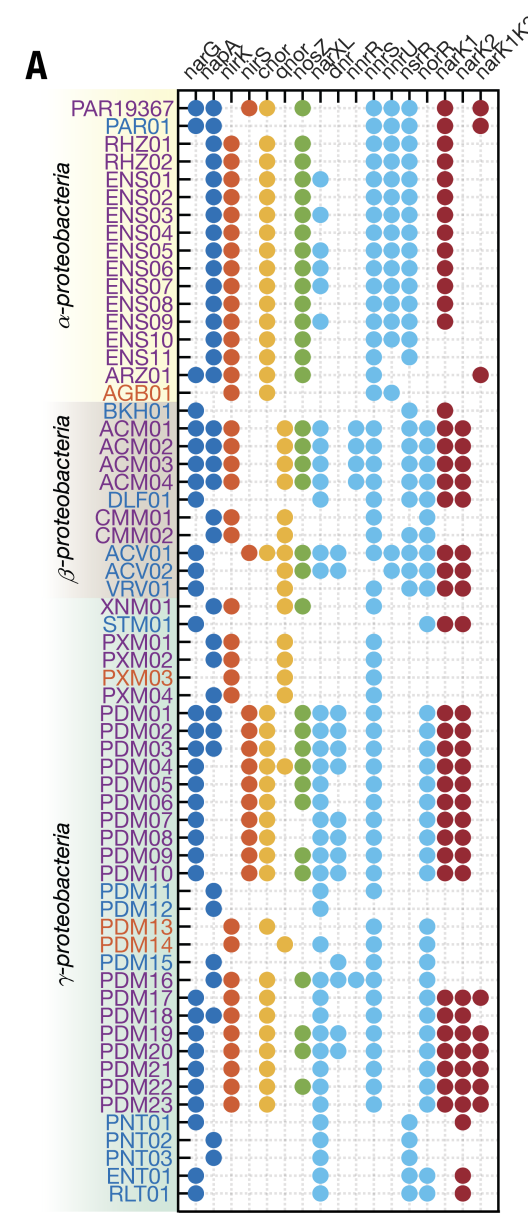

- $\mathrm{NO}_{3}^{-}$reduction $\mathrm{NO}_{2}^{-}$reduction $\mathrm{NO}$ reduction - $\mathrm{N}_{2} \mathrm{O}$ reduction $\odot$ Sensors/regulators - Transporters

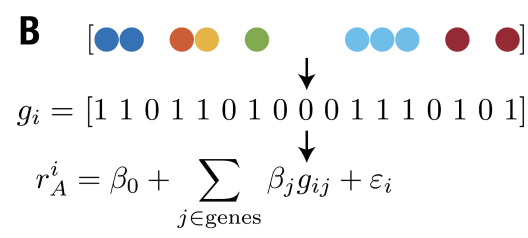

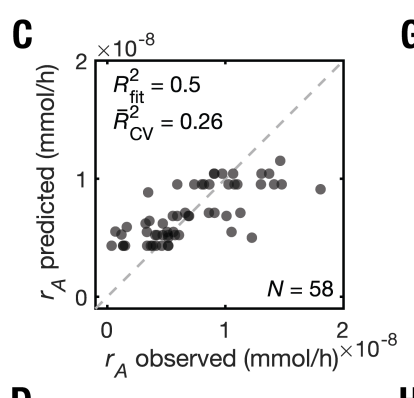
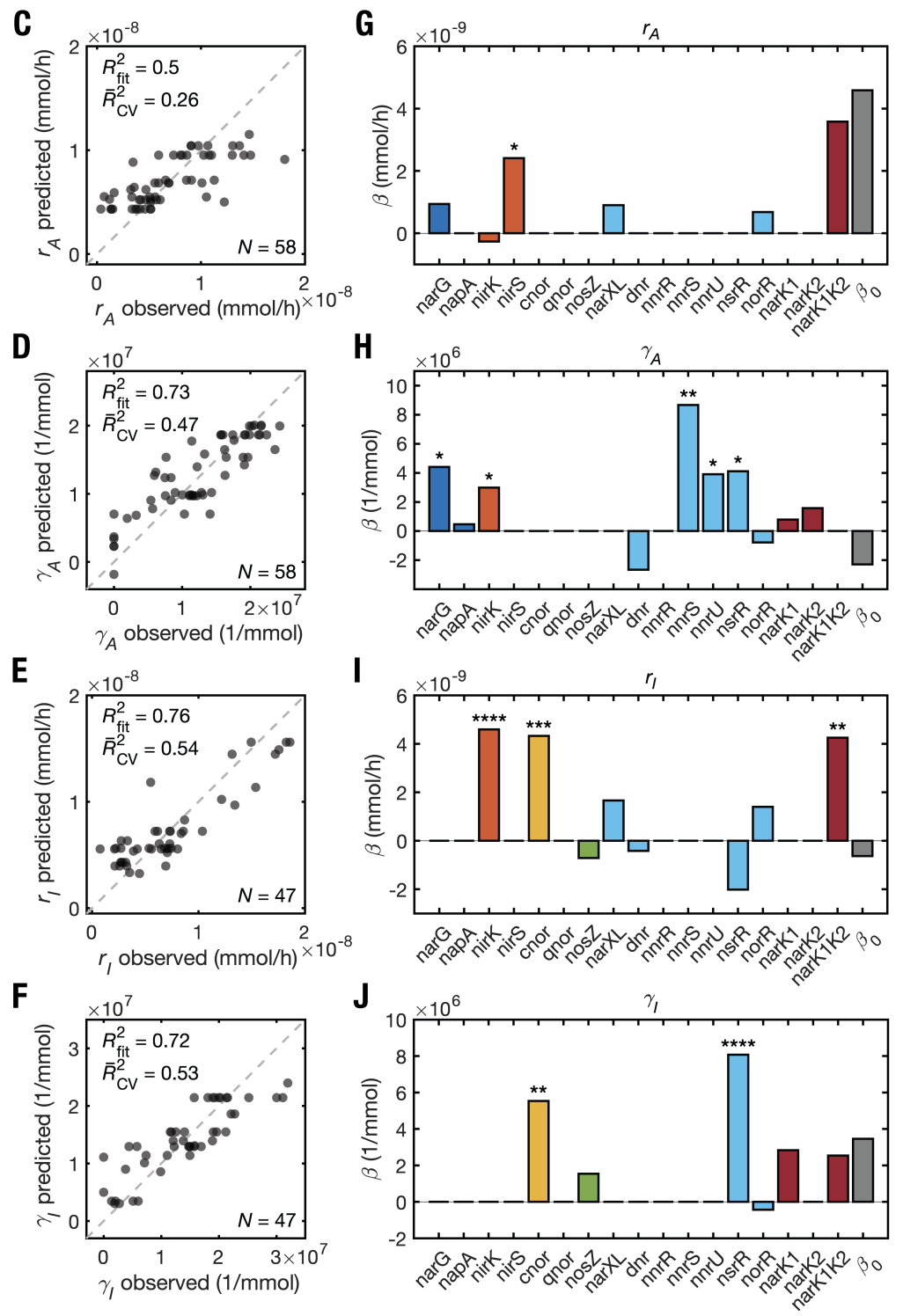

Figure 2: A statistical mapping from gene presence/absence to metabolite dynamics of individual strains. (A) The presence and absence of genes in the denitrification pathway for the 62 denitrifying strains in our library. Strain identifiers correspond to those given in Fig. 1D. The color of each circle corresponds to the known gene function as indicated in the legend below. (B) Observed consumer-resource phenotypic parameters for each strain (e.g., nitrate reduction rate $r_{A}$, Fig. 1D) were linearly regressed against gene presence/absence via $L_{1}$-regularized regression, resulting in regression coefficients $\beta_{j}$ for each gene $j$, an intercept $\beta_{0}$, and a noise term $\epsilon_{i}$ for each observation $i$. Coefficient $\beta_{j}$ captures the impact of possessing gene $j$ on the corresponding phenotypic parameter. Independent regressions were performed for each phenotypic parameter. C, D, E, $\mathbf{F}$ show predicted values of $r_{A}, \gamma_{A}, r_{I}$, and $\gamma_{I}$ respectively plotted against measured values. The dashed line indicates perfect agreement between observations and predictions. The in-sample coefficients of determination for these data $\left(R_{\text {fit }}^{2}\right)$ and the out-of-sample coefficients of determination estimated via iterated cross-validation $\left(\bar{R}_{\mathrm{CV}}^{2}\right)$ are shown. $N$ indicates the number strains in each regression. Strains which do 7 not perform a particular reaction were omitted from the corresponding regression (e.g., Nir strains were excluded from the regression for $r_{A}$ ). $\mathbf{G}, \mathbf{H}, \mathbf{I}, \mathbf{J}$ show estimates of $\beta_{j}$ and $\beta_{0}$ for $r_{A}, \gamma_{A}, r_{I}$, and $\gamma_{I}$ respectively. Asterisks indicate significance level for

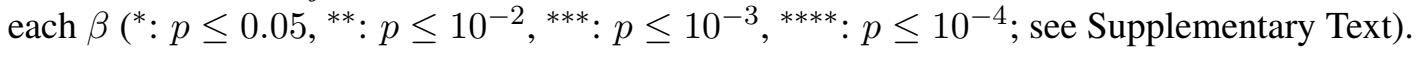


model. LASSO yielded sparse regression models, revealing that presence/absence of a small set of genes is highly predictive of the phenotypic parameters for all strains in our library (Fig. $2 \mathrm{C}$ to $\mathrm{J})$. The in-sample coefficients of determination $\left(R_{f i t}^{2}\right)$ of our regressions were between 0.50 and 0.76 depending on the phenotypic parameter. Crucially, our regression approach generalized out-of-sample, as determined by iterated cross-validation (Supplemental Text, Fig. S9), albeit with slightly lower predictive power $\left(\bar{R}_{C V}^{2}\right.$ between 0.26 to 0.54$)$. Since, in general, traits may exhibit phylogenetic correlation (8), and our library contains a few clades comprising very closely related strains (e.g., ENS01-08, PDM20-23, Fig. 1D), we considered whether our regression utilized phylogenetic correlations in gene presence/absence and denitrification phenotypic parameters to achieve predictive power. We investigated this by collapsing clades containing strains with identical $16 \mathrm{~S}$ rRNA sequences down to a single randomly-selected representative, and performing regressions again on this reduced set of strains. For these regressions we found that the predictive power and coefficients were similar to those for the full dataset (Supplementary Text, Fig. S11), supporting the claim that our regression is not simply detecting phylogenetic correlations between traits and genotypes. Altogether these results demonstrate that, across a diverse set of natural isolates, knowledge of the genes a denitrifying strain possesses is sufficient to accurately predict the rates and biomass yields of that strain on nitrate and nitrite.

Our regression approach leveraged biological knowledge of the denitrification pathway to predict metabolite dynamics, in effect presuming that denitrification gene content is the best genomic feature for prediction. To investigate whether this assumption is correct, we asked whether other genomic properties could better predict metabolite dynamics. First, we tested the predictive capability of sets of randomly selected genes. We chose sets of 17 random genes that were not strongly correlated with any denitrification genes, but retained the same marginal frequency distribution as denitrification genes in the population. We found that regressions using these randomly-selected genes have, on average, much less predictive power than regressions using the denitrification genes (Supplementary Text, Fig. S12). We note that this result provided further evidence that regressions on denitrification gene presence/absence are not simply detecting phylogenetic correlations, since random genes would be expected to perform equally well on average if phenotypes were simply determined by phylogeny. Second, we tested whether $16 \mathrm{~S}$ rRNA copy number or genome size improves the predictive ability of denitrification gene presence/absence regressions. 16S rRNA copy number has been observed to correlate positively with maximal growth rate in nutrient rich conditions $(38,39)$, and smaller genomes are associated with faster growth $(37,39)$. We found that these predictors do not meaningfully alter the regressions or improve their predictive ability (Supplementary Text, Fig. S13 and S14). In summary, our statistical analyses provided evidence that denitrification gene presence/absence outperforms arbitrary sets of genes and coarse genomic features.

Why does gene presence/absence alone hold such strong predictive power for metabolic phenotypes, and why did the regression select specific genes in the denitrification pathway to predict metabolite dynamics? We propose that by quantifying metabolic phenotypes in terms of rates and yields, we captured the salient features of the metabolic process for each strain, which 
allowed the regression to succeed by exploiting the conserved impacts of specific genes on these metabolic phenotypes. To investigate this claim we examined the regression coefficients in the context of what is known about the denitrification pathway. We found that in many cases the sign and magnitude of the regression coefficients agree qualitatively with known properties of the associated enzymes. Previous comparisons between membrane-bound and periplasmic nitrate reductases (encoded by $n a r G$ and napA, respectively) in multiple bacterial species showed that the membrane-bound enzyme exhibits higher nitrate reduction activity in vitro than the periplasmic enzyme (Table S1). This accords with the large positive coefficient for narG we observed in the nitrate reduction rate regression (Fig. 2G). Similarly, in the nitrite reduction rate regression we observed a large positive coefficient for the gene encoding the copper-based nitrite reductase (nirK) (Fig. 2I), which in previous studies showed markedly higher activity in vitro (Table S2) and in vivo (40) compared to the alternate nitrite reductase enzyme encoded by nirS. Further, our regression coefficients showed larger contributions of narG versus napA to yield on nitrate (Fig. 2H), and similarly cnor versus qnor to yield on nitrite (Fig. 2J). Both these observations are consistent with the fact that the genes encoded by narG and cnor contribute more to the proton motive force (and therefore to ATP generation) than their alternatives, napA and qnor, respectively (41). Finally, the transporter encoded by the gene $n a r K 1 K 2$ is a fusion of the nitrate $/ \mathrm{H}^{+}$symporter $\mathrm{NarK} 1$ and the nitrate/nitrite antiporter $\mathrm{NarK} 2$, the latter of which is crucial for exchanging nitrate and nitrite between the cytoplasm and periplasm during denitrification when the membrane-bound nitrate reductase is utilized. In Paracoccus denitrificans, this fusion has been shown to have substantially higher affinity for nitrate than NarK2 alone, resulting in higher growth rates under denitrifying conditions (42). Remarkably this agrees with what we found in the nitrate and nitrite reduction rate regressions, where we observed large positive contributions of narK1K2 (Fig. 2G and I). Taken together, these observations suggest that the regressions exploited mechanistic aspects of the denitrification process to predict metabolite dynamics. However, for many coefficients in our regression, notably regulators, there is no clear interpretation, and definitive proof that these coefficients are mechanistically informative will require genetic manipulation of diverse bacteria.

It is striking that a statistical approach using a library of isolates appears to uncover mechanistic features of the enzymes in the denitrification pathway. This insight was made possible by parameterizing metabolite dynamics with a consumer-resource model that captured the metabolic phenotypes of each strain (rates and yields), and by sampling variation in gene content using a diverse strain library. The approach complements knockout studies in model organisms (43), since it measures the impact of the presence of genes across diverse genetic backgrounds. Our results suggest that this statistical approach could be used to discover the key genomic features of pathways that determine metabolic phenotypes and therefore complement direct genetic interrogation of model organisms. Further, our predictions of metabolic phenotypes from genomes apply across a range of initial conditions and generalized well outof-sample, suggesting that this approach can predict metabolite dynamics in a variety of settings for strains where only genome sequence data are available. 


\section{Predicting metabolite dynamics in communities}

The consumer-resource modeling formalism (Fig. 1C) we used to parameterize metabolite dynamics for each strain allows us to make quantitative predictions for metabolite dynamics in communities of multiple taxa. Since phenotypic parameters were sparsely encoded by the genomes of each strain (Fig. 2), predicting community metabolite dynamics from the consumerresource model would provide a direct mapping from gene content to community metabolism. Therefore, we extended to our modeling formalism to $N$-strain communities by adding the rate contributions of each strain to the dynamics of nitrate and nitrite (Fig. 3B, Supplementary Text). This model assumes that strains interact only via cross-feeding and resource-competition for electron acceptors. This "additive" model also assumes that the rates and yields on nitrate and nitrite for strains in pair-culture are the same as in monoculture. As a result, the model provides predictions for $N$-strain community metabolite dynamics without any free parameters.

We first tested the ability of this approach to predict metabolite dynamics in all pair combinations of 12 strains from our library ( 4 Nar/Nir, 4 Nar, 4 Nir). We assembled communities in 96-well plates containing chemically-defined medium and sampled over a $64 \mathrm{~h}$ period to measure concentrations of nitrate and nitrite (Materials and Methods). Remarkably, we found that the additive model accurately predicted the metabolic dynamics for most 2 -strain communities (Fig. 3, Fig. S15 and S16). Specifically, the third column of Fig. 3A shows the zero-freeparameter predictions (lines) of denitrification dynamics in 2-strain communities, which agreed well with measurements (points). The 2-strain community predictions include non-trivial dynamics such as two Nar strains exhibiting faster nitrate reduction as a collective or a transient increase nitrite in a Nar/Nir + Nar community.

We quantified the quality of the additive model predictions by computing a normalized root-mean-square error (NRMSE, see caption of Fig. 3). NRMSE in the range 0-2 indicates predictions in 2-strain communities that are similar in quality to the fits of the constituent monocultures. We found that most 2-strain communities have low NRMSE, indicating that our model successfully predicted metabolite dynamics in most cases, given only knowledge of the monoculture rates and yields for each strain. The success or failure of the model depended on the phenotypes of the strains present. The model successfully predicted 2-strain metabolite dynamics for most types of communities (e.g., Nar/Nir + Nar or Nar + Nar) but failed only in the case where Nar strains were cultured with Nir strains (Fig. 3A and C, Fig. S17). We speculate that the failure of the model to predict metabolite dynamics in Nar + Nir communities was caused by excretion of nitric oxide by the Nir strain, which can be cytotoxic to strains that do not express nitric oxide reductase (44), and may consequently slow Nar strain growth. Although both Nar/Nir and Nir strains are capable of generating extracellular nitric oxide, Nir isolates have been observed in a previous study to transiently generate nitric oxide at higher concentrations (31), possibly explaining why the 2 -strain additive model fails only to predict $\mathrm{Nar}+\mathrm{Nir}$ communities.

We next asked whether information from monocultures also successfully predicted metabolite dynamics in 3-strain communities. We applied the additive model to predicting the nitrate 
A
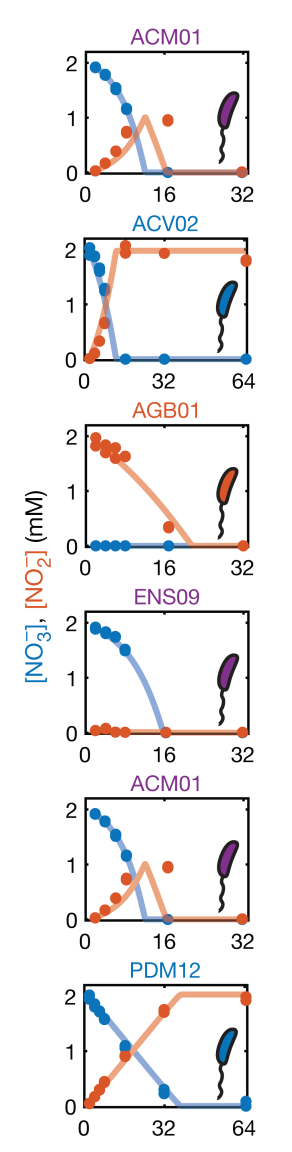
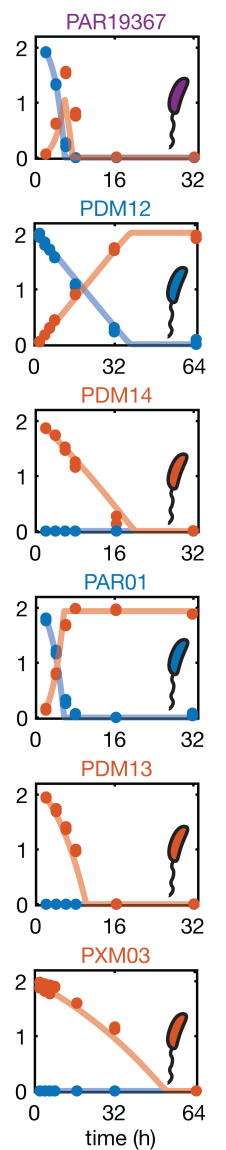

zero-free-parameter $\mathbf{B}$ predictions

ACM01 + PAR19367

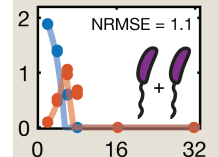

ACV02 + PDM12
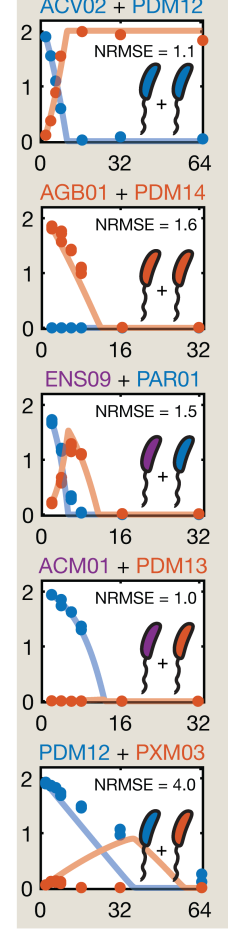

$$
\begin{aligned}
\frac{d x_{i}}{d t} & =\left(\gamma_{A} r_{A}^{i} \frac{A}{K_{A}+A}+\gamma_{I}^{i} r_{I}^{i} \frac{I}{K_{I}+I}\right) x_{i} \\
\frac{d A}{d t} & =-\sum_{i=1}^{N} r_{A}^{i} \frac{A}{K_{A}+A} x_{i} \\
\frac{d I}{d t} & =\sum_{i=1}^{N}\left(r_{A}^{i} \frac{A}{K_{A}+A}-r_{I}^{i} \frac{I}{K_{I}+I}\right) x_{i} .
\end{aligned}
$$

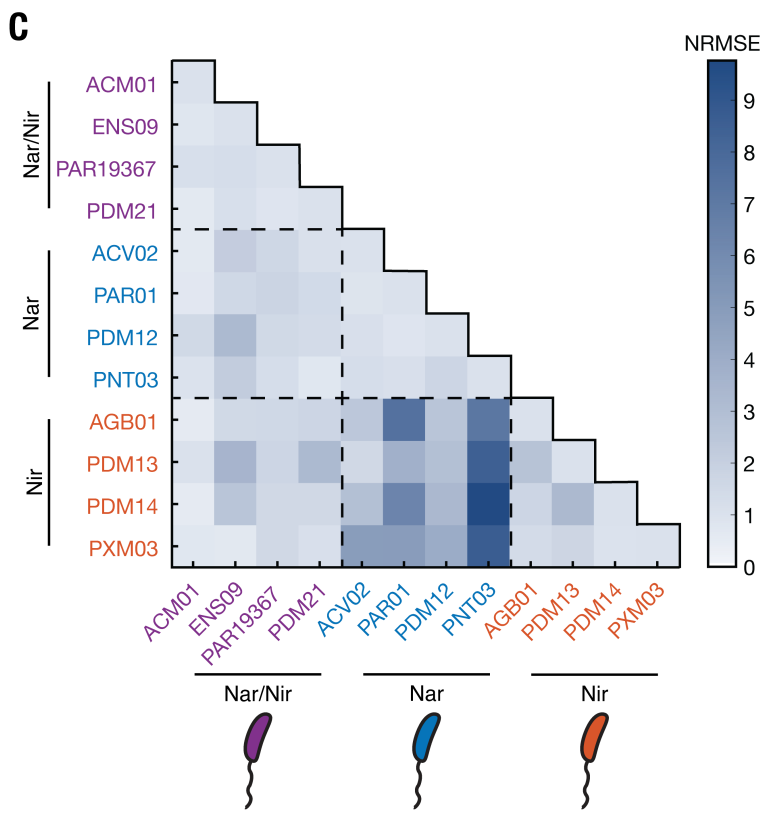

Figure 3: Metabolite dynamics in two-strain communities are predictable from monocultures. (A) Examples of pair-culture dynamics for all combinations of the three denitrification phenotypes (Nar/Nir, purple; Nar, blue; Nir; red). The first two columns show metabolite dynamics for each of two strains cultured individually. The third column shows the metabolite dynamics for pair-cultures of the two strains (points) with zero free parameter predictions using the consumer-resource model (curves, see panel $\mathbf{B}$ for model). Errors in pair-culture predictions are shown in each panel in the third column as quantified by the normalized root-mean-square error (NRMSE). We define $N R M S E_{i j}=$ $R M S E_{i j} / \sqrt{\left(R M S E_{i}^{2}+R M S E_{j}^{2}\right) / 2}$, where $\mathrm{RMSE}_{i j}$ is the root-mean-square error between model predictions and observed metabolite concentrations of strains $i$ and $j$ in pair-culture, and $\mathrm{RMSE}_{i}$ and $\mathrm{RMSE}_{j}$ are the RMSEs of strains $i$ and $j$ in monoculture. NRMSE values between 0 and 2 indicate predictions of pair-culture metabolite dynamics of similar quality to the corresponding monocultures. (B) An $N$-strain consumer-resource model (based on Fig. 1C) was used to predict pair-culture metabolite dynamics $(N=2) . A$ and $I$ are nitrate and nitrite concentrations respectively. $x_{i}$ denotes abundance of strain $i$ with parameters $r_{A}^{i}, \gamma_{A}^{i}, r_{I}^{i}$ and $\gamma_{I}^{i}$, which were determined from monoculture experiments (Fig. 1D). The $K_{*}$ values are fixed for all strains. (C) A matrix of NRMSE values quantifying the quality of model predictions for all pairs of 12 strains: 4 Nar/Nir, 4 Nar, 4 Nir as indicated left and below. Strain names correspond to Figs. 1D and 2A. Only Nar + Nir communities are poorly predicted by the consumer-resource model ( $p<10^{-5}$, Fig. S16, Figure 4). 
and nitrite dynamics in 81 random combinations of 3 strains from the 12-strain subset. In communities that did not contain a Nar + Nir pair (e.g., Fig. 4A), we found that prediction accuracy was high (grey points, Fig. 4B, Fig. S18). This again indicated that in most combinations of phenotypes, community dynamics were predictable from consumer-resource parameters for each strain in the community. However, in communities that contained a Nar + Nir pair, predictions were relatively poor (yellow points, Fig. 4B, Fig. S18), suggesting that interactions between Nar and Nir phenotypes that were not captured in the additive model were again driving low prediction accuracy.

To address the impact of interactions between Nar and Nir strains not accounted for by our additive model in 3-strain communities, we took a coarse-graining approach. We asked whether the collective metabolism of Nar + Nir pairs could be treated as modules within 3strain communities. To accomplish this we re-fitted nitrate and nitrite reduction rates $\left(r_{A}, r_{I}\right)$ to pair-culture data for each Nar + Nir pair, leaving yields fixed (Fig. 4C, Supplementary Text, Fig. S19). This resulted in effective nitrate and nitrite reduction rates $\left(\tilde{r}_{A}, \tilde{r}_{I}\right)$ for each Nar + Nir pair. We then used these rates to make predictions for 3-strain communities that included a $\mathrm{Nar}+$ Nir pair (e.g., Fig. 4D). For 3-strain communities that included multiple Nar + Nir pairs (e.g., Nar + Nar + Nir), we developed simple rules for determining the effective rates from the rates for each Nar + Nir pair present (Supplementary Text). We found that the metabolite dynamics in 3-strain communities containing Nar + Nir pairs were quantitatively predicted by this coarse-graining approach (yellow points, Fig. 4B). We conclude that treating Nar + Nir pairs as effective modules within larger communities recovers the predictive power of the additive consumer-resource model.

\section{Discussion}

Quantifying the metabolic phenotypes of a diverse library of natural isolates using a consumerresource model allowed us to take a statistical approach to connecting genotypes to dynamical metabolic phenotypes. The outcome was a sparse mapping from gene content to metabolite dynamics for each strain in the library that appears to exploit known mechanistic features of genes in the denitrification pathway to achieve predictive power. The resource-based modeling formalism permitted quantitative predictions of community-level metabolite dynamics. As a result, the approach yielded a direct mapping from genomic structure to metabolite dynamics at the community-level.

At the cellular level, the apparent mechanistic relevance of the regression coefficients in this study suggests that a statistical approach, coupled with large-scale culturing and phenotyping on libraries of isolates $(45,46)$, could be exploited to discover the salient features of genomes that determine other metabolic functions. Higher throughput measurements should enable a more detailed interrogation of genomic features, allowing us to extend our statistical approach to gene sequences, promoter architecture, and synteny. These insights might then be used to design genomes and communities with predefined metabolic capabilities by the addition or 

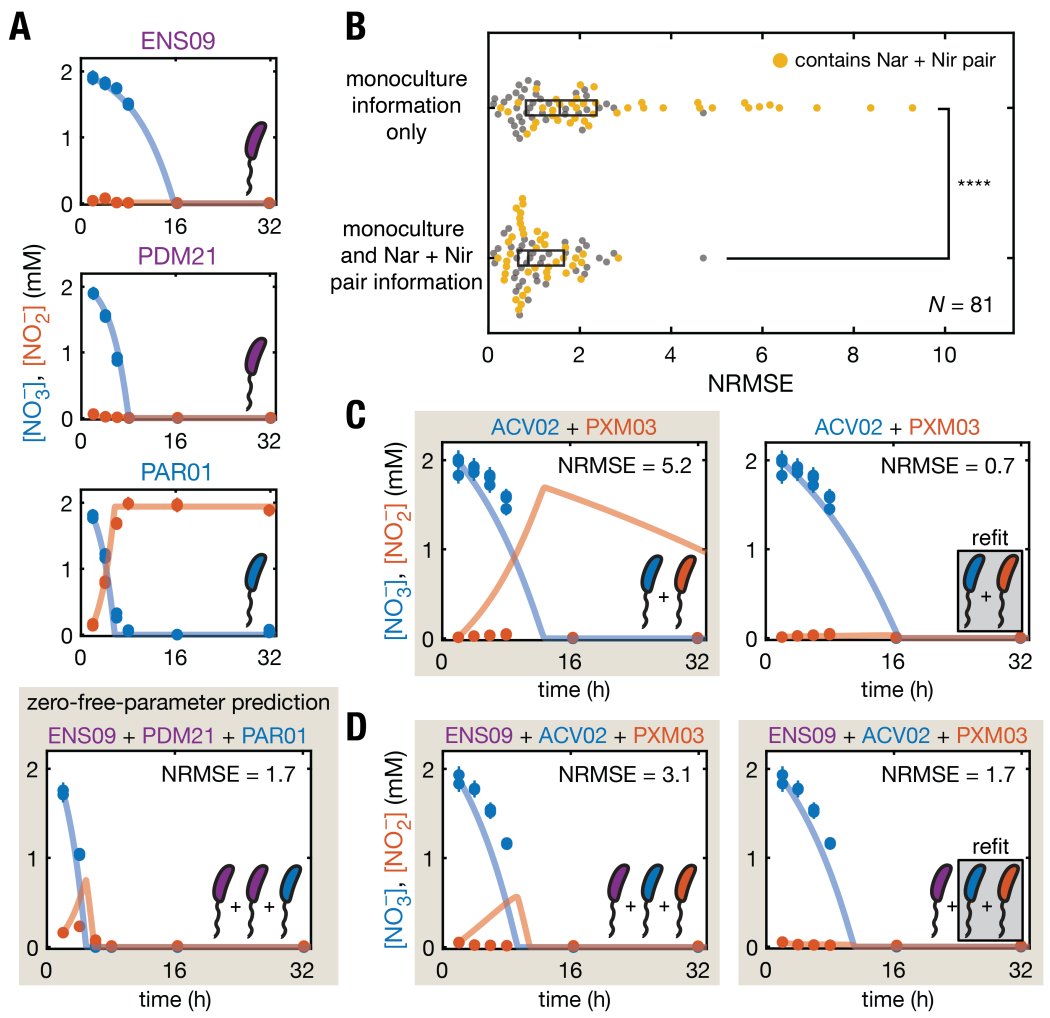

Figure 4: Metabolite dynamics in 3-strain communities are predictable. The consumer-resource model provides predictions for metabolite dynamics in communities of three strains, and these predictions were verified experimentally. (A) Metabolite dynamics for an example 3-strain ( $\mathrm{Nar} / \mathrm{Nir}+\mathrm{Nar} / \mathrm{Nir}$ + Nar) community. The first three panels show metabolite dynamics for each strain cultured individually, and the fourth panel shows the metabolite dynamics of the 3-strain community. Curves show the prediction of the consumer-resource model (Fig. 3B). (B) NRMSE (see Fig. 3 caption) values quantifying quality of consumer-resource model predictions for 3-strain communities. Orange and (gray) points denote 3-strain communities that do (do not) contain a Nar + Nir pair. Nar + Nir pair-culture dynamics are poorly predicted by the model (Fig. 3C) and result in high NRMSE in 3-strain communities containing Nar + Nir pairs (compare orange and gray points). Top and bottom scatter plots compare predictions from a consumer-resource model using only monoculture data to a coarse-graining approach that describes Nar + Nir pairs as modules within the 3-strain community (described in C-D). The coarsegraining approach improves the 3 -strain community predictions ( $t$-test, ${ }^{* * * *}$ denotes $\left.p=7 \times 10^{-6}\right)$. $(\mathbf{C})$ Metabolite dynamics for an example Nar + Nir pair, where curves in the left panel show the prediction of the consumer-resource model using only parameters fit to monocultures, and curves in the right panel show the results of refitting the reduction rates $\left(r_{A}, r_{I}\right)$ to Nar + Nir pair-culture data but leaving yields $\left(\gamma_{A}, \gamma_{I}\right)$ fixed to monoculture values. (D) Metabolite dynamics for a 3-strain community containing a Nar/Nir strain and the Nar + Nir pair shown in panel C. Curves in the left panel show the prediction of the consumer-resource model using parameters inferred from monoculture experiments for each strain, and curves in the right panel show the prediction when the Nar + Nir pair is treated as a module with rate parameters refit from pair-culture data (right panel in C). Note the reduction in NRMSE due to the coarse-graining of the Nar + Nir pair. Panels in beige denote zero free parameter predictions. 
deletion of specific genes (47).

At the community-level our approach could eventually enable the prediction of metabolite dynamics in complex communities where functional gene content has been assigned to individual genomes (48). Soils and host-associated communities typically contain thousands of bacterial taxa, so testing the predictive power of the consumer-resource formalism in communities of many taxa in more complex environments will be essential. However, micron-scale spatial structure in soils suggests that denitrification may occur locally, in communities of just a few taxa (49), meaning that the rules of denitrification for simple communities could help us understand more complex contexts. A predictive understanding of denitrification in complex communities is key for minimizing harmful nitrous oxide emissions (50) and controlling bacterial nitric oxide production in mammalian hosts $(51)$.

The evolutionary and ecological basis for our mapping from genomic structure to community function remains to be discovered, but our results lend support to the idea that different genes in the denitrification pathway are adapted to different ecological niches $(21,30)$. Combining our understanding of how gene content determines phenotype with analyses of horizontal gene transfer and community assembly in specific niches could yield insights into how evolutionary and ecological processes combine to shape community structure and function.

\section{References}

1. M. J. Follows, S. Dutkiewicz, S. Grant, S. W. Chisholm, Science 315, 1843 (2007).

2. H. Lu, K. Chandran, D. Stensel, Water Research 64, 237 (2014).

3. S. Subramanian, et al., Nature 510, 417 (2014).

4. K. Anantharaman, et al., Nature Communications 7, 13219 (2016).

5. N. Molina, E. van Nimwegen, Trends in Genetics 25, 243 (2009).

6. I. Sela, Y. I. Wolf, E. V. Koonin, Physical Review X 9, 031018 (2019).

7. W. Shou, S. Ram, J. M. G. Vilar, Proceedings of the National Academy of Sciences of the United States of America 104, 1877 (2007).

8. J. B. H. Martiny, S. E. Jones, J. T. Lennon, A. C. Martiny, Science 350 (2015).

9. E. E. Lilja, D. R. Johnson, The ISME Journal 10, 1568 (2016).

10. D. M. Ward, et al., Philosophical Transactions of the Royal Society B: Biological Sciences 361, 1997 (2006).

11. O. X. Cordero, L.-A. Ventouras, E. F. DeLong, M. F. Polz, Proceedings of the National Academy of Sciences 109, 20059 (2012). 
12. A. Sanchez-Gorostiaga, D. Bajić, M. L. Osborne, J. F. Poyatos, A. Sanchez, PLOS Biology 17, e3000550 (2019).

13. H. Mickalide, S. Kuehn, Cell Systems 9, 521 (2019).

14. N. Klitgord, D. Segrè, Current Opinion in Biotechnology 22, 541-546 (2011).

15. M. Mori, T. Hwa, O. C. Martin, A. D. Martino, E. Marinari, PLOS Computational Biology 12, e1004913 (2016).

16. W. Harcombe, et al., Cell Reports 7, 1104 (2014).

17. C. J. Norsigian, X. Fang, Y. Seif, J. M. Monk, B. O. Palsson, Nature Protocols 15, 1 (2020).

18. S. Louca, et al., Nature Ecology \& Evolution 2, 936 (2018).

19. C. Huttenhower, et al., Nature 486, 207 (2012).

20. C. Burke, P. Steinberg, D. Rusch, S. Kjelleberg, T. Thomas, Proceedings of the National Academy of Sciences 108, 14288 (2011).

21. C. M. Jones, S. Hallin, The ISME Journal 4, 633-641 (2010).

22. N. Fierer, et al., The ISME Journal 6, 1007 (2012).

23. S. Louca, L. W. Parfrey, M. Doebeli, Science 353, 1272 (2016).

24. J. E. Goldford, et al., Science 361, 469 (2018).

25. M. S. Datta, E. Sliwerska, J. Gore, M. F. Polz, O. X. Cordero, Nature Communications 7 (2016).

26. J. Friedman, L. M. Higgins, J. Gore, Nature Ecology \& Evolution 1, 1 (2017).

27. W. G. Zumft, Microbiology and Molecular Biology Reviews 61, 84 (1997).

28. S. Seitzinger, et al., Ecological Applications 16, 2064-2090 (2006).

29. T. Irrazabal, A. Belcheva, S. E. Girardin, A. Martin, D. J. Philpott, Molecular Cell 54, 309 (2014).

30. D. R. H. Graf, C. M. Jones, S. Hallin, PLoS ONE 9, e114118 (2014).

31. P. Lycus, et al., The ISME Journal 11, 2219 (2017).

32. K. Heylen, et al., Environmental Microbiology 8, 2012 (2006). 
33. C. M. Jones, B. Stres, M. Rosenquist, S. Hallin, Molecular Biology and Evolution 25, 1955 (2008).

34. A. F. Schober, et al., Cell Reports 27, 3359 (2019).

35. D. A. Rodionov, I. L. Dubchak, A. P. Arkin, E. J. Alm, M. S. Gelfand, PLoS Computational Biology 1, 17 (2005).

36. J. W. B. Moir, N. J. Wood, Cellular and Molecular Life Sciences 58, 215-224 (2001).

37. M. Lynch, Annual Review of Microbiology 60, 327 (2006).

38. B. R. K. Roller, S. F. Stoddard, T. M. Schmidt, Nature Microbiology 1, 16160 (2016).

39. J. Li, et al., The ISME Journal 13, 2162 (2019).

40. A. B. Gloekner, A. Jiingst, W. G. Zumft, Archives of Microbiology 160, 18-26 (1993).

41. S. J. Ferguson, D. J. Richardson, The Enzymes and Bioenergetics of Bacterial Nitrate, Nitrite, Nitric Oxide and Nitrous Oxide Respiration (Springer, 2004), vol. 2, p. 169-206.

42. A. D. Goddard, J. W. B. Moir, D. J. Richardson, S. J. Ferguson, Molecular Microbiology 70, 667-681 (2008).

43. R. J. Nichols, et al., Cell 144, 143 (2011). Publisher: Elsevier.

44. C. Braun, W. G. Zumft, The Journal of Biological Chemistry 266, 22785-22788 (1991).

45. S. A. Connon, S. J. Giovannoni, Applied and Environmental Microbiology 68, 3878 (2002).

46. J. Kehe, et al., Proceedings of the National Academy of Sciences 116, 12804 (2019).

47. A. J. Shaw, et al., Proceedings of the National Academy of Sciences 105, 13769 (2008).

48. C. M. K. Sieber, et al., Nature Microbiology 3, 836 (2018).

49. R. Lensi, A. Clays-Josserand, L. Jocteur Monrozier, Soil Biology and Biochemistry 27, 61 (1995).

50. M. A. Cavigelli, G. P. Robertson, Ecology 81, 1402 (2000).

51. E. R. Hyde, et al., PLOS ONE 9, e88645 (2014). 


\section{Acknowledgments}

We thank Laura Troyer for assistance with isolating bacterial strains, and Elizabeth Ujhelyi and Annette Wells for assistance with sequencing. We acknowledge Cameron Pittelkow for access to corn and soybean fields in Savoy, Illinois, and the laboratory of Julie Zilles for providing the bacterial strain Paracoccus denitrificans ATCC 19367. We also thank Rama Ranganathan, David Pincus, James Sethna, William Metcalf, Jun Song and members of the Kuehn laboratory and Mani group for helpful discussions. Funding: This work was supported by the National Science Foundation Physics Frontiers Center Program PHY 0822613 and PHY 1430124 (S.K.), James S. McDonnell Foundation Postdoctoral Fellowship Award \#220020499 (K.G.), and the Simons Foundation Investigator Award \#597491 (M.M.). Authors contributions: K.G.: Conceptualization, experimental design, data collection, formal analysis, coding, writing - original draft. D.P.: data collection. M.M: Conceptualization, formal analysis, writing - revision \& editing, supervision, funding acquisition. S.K.: Conceptualization, experimental design, formal analysis, writing - original draft, supervision, funding acquisition. Competing interests: The authors declare no competing interests. Data and materials availability: Draft genome assemblies are deposited on NCBI (BioProject PRJNA660495). Annotation files and data used in the regressions are deposited on Open Science Framework (doi: 10.17605/OSF.IO/T3PRD). Isolates are available upon request.

\section{Supplementary materials}

Materials and Methods

Supplementary Text

Figs. S1 to S19

Tables S1 to S9

References (52-92) 